\title{
Needle Steering System using Duty-Cycled Rotation for Percutaneous Kidney Access
}

\author{
Nathan A. Wood, Khaled Shahrour, Michael C. Ost, and Cameron N. Riviere
}

\begin{abstract}
The authors present ongoing work on the use of a variable curvature flexible needle steering system to gain percutaneous access to the kidney for medical interventions. A nonlinear control law is introduced which drives the needle to track a predetermined planar path using a steering approach based on duty-cycled rotation during insertion. Renal access is performed in simulation and tested in vitro in a tissue phantom to validate the proposed control method.
\end{abstract}

\section{INTRODUCTION}

Percutaneous renal access is important for multiple clinical interventions including urinary drainage, management of renal tumors during biopsy, and treatment of stones. The most important step in percutaneous renal procedure is to have an appropriate renal access that meets the goals of the surgery. Presently, however, percutaneous renal access has multiple limitations. Percutaneous access for nephrolithotomy has a steep learning curve that limits its use among urologists despite the proven benefits of an access created by urologists compared to that created by interventional radiologists [1,2]. Even with extensive experience, percutaneous renal access can be difficult to obtain in many instances when the tumor is small or intraparenchymal, or when the kidney is abnormally positioned or oriented.

Percutaneous access carries the risk of injuring adjacent organs such as the lungs, colon, liver, spleen, and duodenum [3]. Moreover, radiation exposure to the operating surgeon during percutaneous nephrolithotomy has been estimated to be highest in comparison to other endourological procedures; the amount of fluoroscopy time used is greater by a factor of 10 [4]. These disadvantages motivate the search for alternatives to the standard clinical approach, which is based on rigid needles and fluoroscopic guidance. A flexible needle that can be guided predictably and precisely to a target in the kidney may simplify the procedure and decrease exposure to radiation.

Robotic techniques for control of needle insertion, and more specifically for steering of flexible needles, have been a subject of active research in recent years [5]. Among the approaches that have been developed is one that exploits the natural deflection of a bevel-tip needle during insertion into soft tissue, controlling the trajectory of the needle by

This work was supported in part by the Simeon M. Jones, Jr. and Katharine Reed Jones Fund of The Pittsburgh Foundation.

N. A. Wood and C. N. Riviere are with the Robotics Institute, Carnegie Mellon University, Pittsburgh, PA 15213 USA (e-mail: camr@ri.cmu.edu).

K. Shahrour and M. C. Ost are with the Department of Urology, University of Pittsburgh, Pittsburgh, PA 15213 USA. adjusting the orientation of the bevel during insertion [6]. We have extended this approach by adding a means for proportional control of curvature [7]. The present paper describes a technique for closed-loop control of a needle steered after the manner of [7], in order to follow a preoperatively planned path for renal access. Results are presented from simulation and from testing in vitro in a tissue phantom.

\section{METHODS}

\section{A. Needle Steering via Duty-Cycled Rotation}

Duty-cycled rotation of a bevel tipped needle allows for proportional control of the curvature of bevel tipped needles during insertion. This method works by alternating between periods of (i) insertion without rotation, in which the needle follows a path of maximum curvature, and (ii) insertion with rotation, in which the needle moves straight. Assuming constant velocities, duty cycle, $D C$, is defined as the ratio of the rotation period, $\tau_{\text {rot }}$, to the cycle period, $T$, where the cycle period is the sum of the rotation period and the translation period, $\tau_{\text {trans }}$.

$$
D C=\frac{\tau_{\text {rot }}}{T}=\frac{\tau_{\text {rot }}}{\tau_{\text {rot }}+\tau_{\text {trans }}}
$$

The effective curvature, $\kappa$, of the path a needle follows under duty-cycled spinning is then defined as linear function of the duty cycle and the maximum nominal curvature, $\kappa_{\text {max }}$, of the needle-tissue combination.

$$
\kappa=\kappa_{\max }(1-D C)
$$

Using the relationship in (2) the needle can be driven to follow a path of any curvature up to the maximum value [7].

Implementation of this method of needle steering is accomplished by moving a fixed distance each cycle, $\Delta s$, and having a fixed rotational velocity, $\omega$. A motion cycle is then fully defined by

$$
\tau_{\text {rot }}=\frac{2 \pi}{\omega}, \tau_{\text {trans }}=\frac{\tau_{\text {rot }}(1-\mathrm{DC})}{\mathrm{DC}}, u=\frac{\Delta s}{T}
$$

where $u$ is translational velocity.

\section{B. Path Tracking}

Duty-cycled control, in conjunction with paths that have curvatures smaller than the maximum curvature of the needle, allows for path tracking to be implemented. By 
constraining the normal of the needle-tip bevel to a plane when not rotating, the system can be approximated with a planar model. Previous work has shown that the kinematics of the needle tip as it is inserted into tissue can be modeled as a bicycle in which the needle tip is located between the front and rear wheels, and less accurately as a unicycle with constrained angular velocity [6]. At this stage of the work, for simplicity in implementation, a unicycle model is used to describe the motion of the needle tip.

$$
\frac{d}{d s}\left[\begin{array}{l}
x \\
y \\
\theta
\end{array}\right]=\left[\begin{array}{c}
\cos \theta \\
\sin \theta \\
\kappa
\end{array}\right]
$$

The tracking control law implemented here is based on the Stanley method [8], which was used by Stanford University's DARPA Grand Challenge autonomous vehicle. The nonlinear function steers the front wheel of a kinematic bicycle model, $\phi$, to correct for heading error, $\theta_{e}$, and crosstrack error, $e_{c t}$, where $k$ is a gain parameter.

$$
\phi(t)=\theta_{e}(t)+\tan ^{-1} \frac{k e_{c t}(t)}{v_{x}(t)}
$$

Slight modifications are made to this control law for implementation on the unicycle model. In order to directly control the curvature of the unicycle model, the steering angle from (5) is converted to curvature using the relationship

$$
\kappa=\mathrm{k}_{2} \tan \phi
$$

where, for a bicyle model the parameter $\mathrm{k}_{2}$ is the reciprocal of the wheelbase, but in this case is considered a tuning parameter. The Stanley method defines crosstrack error as the distance from the front wheel to the nearest point on the desired path, which will be considered to be the distance from the needle tip to the nearest point on the path. Because of the nature of the duty-cycled rotation technique, the velocity term in the control law, $v_{x}$, is replaced by distance per cycle, $\Delta s$.

$$
\kappa=k_{2} \tan \left(\theta_{e}+\tan ^{-1} \frac{k_{1} e_{c t}}{\Delta s}\right)
$$

\section{Experimentation}

In order to test the proposed control system, shown in Fig. 1, simulation and in vitro tests were conducted. Start and goal configurations were defined using a medical training model (\#60502 Percutaneous Nephrolithotomy Slab, Limbs \& Things, Inc., Savannah, Ga.), which was used as a tissue phantom for the test in vitro. The goal positions were set at the centers of minor calyces with the orientation parallel with the blood vessels.

The needle used in the experiment was a $0.52 \mathrm{~mm}$ diameter nitinol wire with a bevel of $7^{\circ}$. The maximum nominal curvature, $\kappa_{\max }$, of this needle-tissue combination was $0.003 \mathrm{~mm}^{-1}$. Tuning of the control parameters was done in simulation. Control gains used for both tests were $k_{1}=1.0$ and $k_{2}=0.01$. Rotational velocity, $\omega$, was set to 1 $\mathrm{rev} / \mathrm{s}$, and step size, $\Delta s$, was set to $1 \mathrm{~mm}$.

1) Simulation Model: The 3D model for a needle under duty-cycled spinning used for simulation was previously shown in [7].

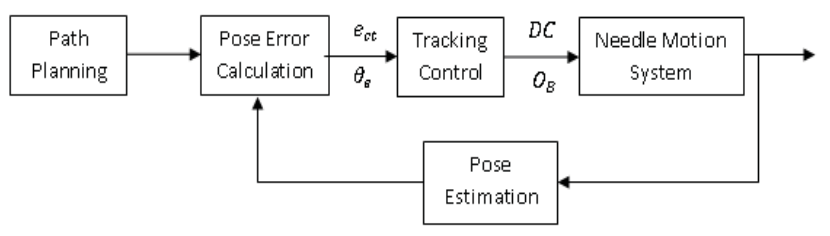

Figure 1. Experimental system control loop.

2) Path Planning: Planar trajectories for the needle are calculated in a manner similar to the inverse kinematic method presented in [9]. The major difference is that in the case of $100 \%$ duty cycle the path has zero curvature, allowing for Dubins paths [10]. The maximum curvature of the planned paths was $0.0016 \mathrm{~mm}^{-1}$, or approximately half of the maximum nominal curvature, $\kappa_{\max }$.

3) Pose Estimation: An Extended Kalman Filter (EKF) is used to estimate the state of the needle. The system model implemented is the unicycle model given in (4). Needle tip location in $2 \mathrm{D}$ is measured using image processing of video from a camera (Dragonfly, Point Grey Research, Inc., Richmond, BC) mounted $25 \mathrm{~cm}$ directly above the tissue phantom and assumed to be normal to the plane in which the needle operates. Prior to introduction of the needle into the tissue, a background image is initialized. This background image is then subtracted from each image taken during testing leaving only the needle as the image foreground. The position of the needle tip is then determined by finding the distal most point of the foreground object. Estimates of the needle heading are determined through the process model of the EKF. In simulation, perfect sensors are assumed.

\section{Results}

Results from a simulation of the needle system are shown in Figs. 2 and 3. The simulated needle was initialized with a $2.5 \mathrm{~mm}$ cross track error from the desired path. The implemented control law drives the needle towards the path with maximum positive curvature for approximately $25 \mathrm{~mm}$ eliminating the initial error and then smoothly controls the curvature of the needle to follow the pre-planned path as desired.

Commanded duty cycle for the simulation is shown in Fig. 3. In the plot the vertical duty cycle axis ranges from zero at the top, corresponding to maximum positive curvature, to zero at the bottom, corresponding to maximum negative curvature, which both occur at a duty cycle of zero. The transition from positive to negative curvature occurs in the plot as the trace crosses a duty cycle of 1.0, corresponding to zero curvature. A change in sign of 
curvature is accomplished by rotating the needle $180^{\circ}$ about its axis.
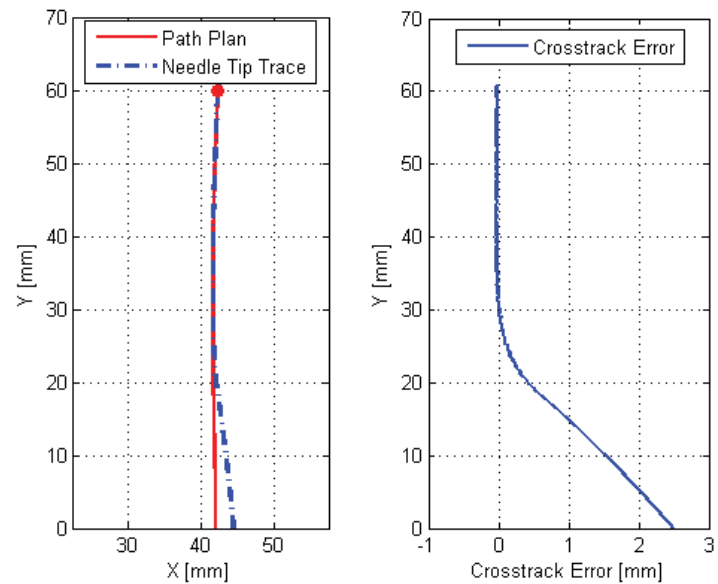

Figure 2. Simulated path tracking trace (left) and crosstrack error (right). Crosstrack error is the deviation of the needle tip from the path.

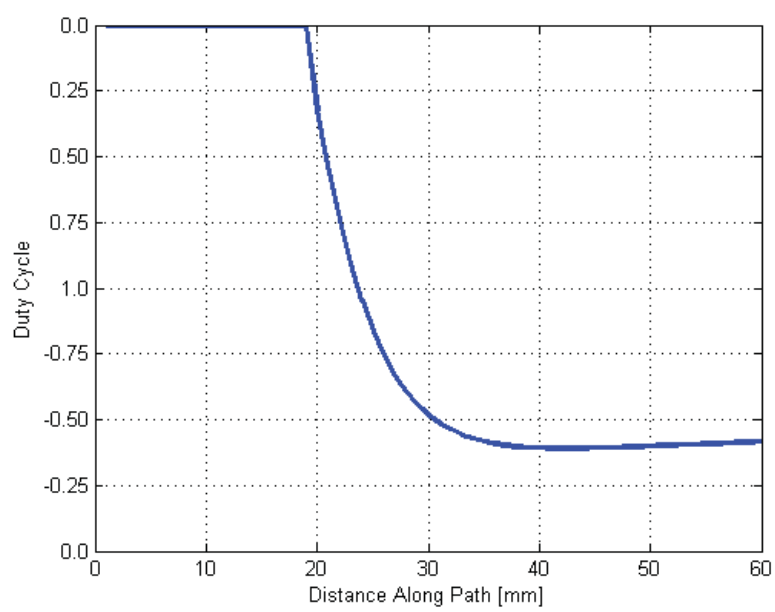

Figure 3. Simulated path tracking control duty cycle output. Duty cycles corresponding to maximum positive and negative curvature are located at the top and bottom of the vertical axis respectively. As the trace crosses a

duty cycle of 1.0 curvature transitions from positive to negative.

Results from in vitro testing of the needle system are shown in Figs. 4-6. Using the proposed method, the needle successfully entered the phantom kidney through a minor calyx. The in vitro performance differed somewhat from simulated results specifically when comparing the crosstrack errors. Initially the experimental duty cycle matches the simulation results commanding maximum positive curvature to eliminate cross track error, but the needle did not make as smooth of a transition to following the planned path as in simulation. The control law was able to follow the desired path reasonably well, with an average crosstrack error magnitude of $0.71 \mathrm{~mm}$ and a maximum of $1.44 \mathrm{~mm}$.

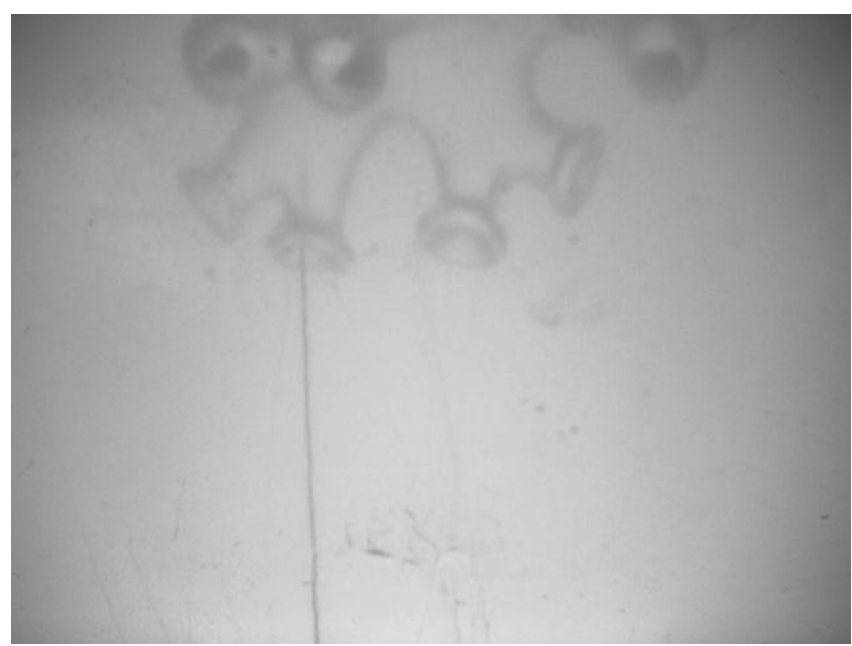

Figure 4. Final result from test in vitro in kidney tissue phantom. The needle tip is visible inside one of the calyces of the kidney at the top of the figure.
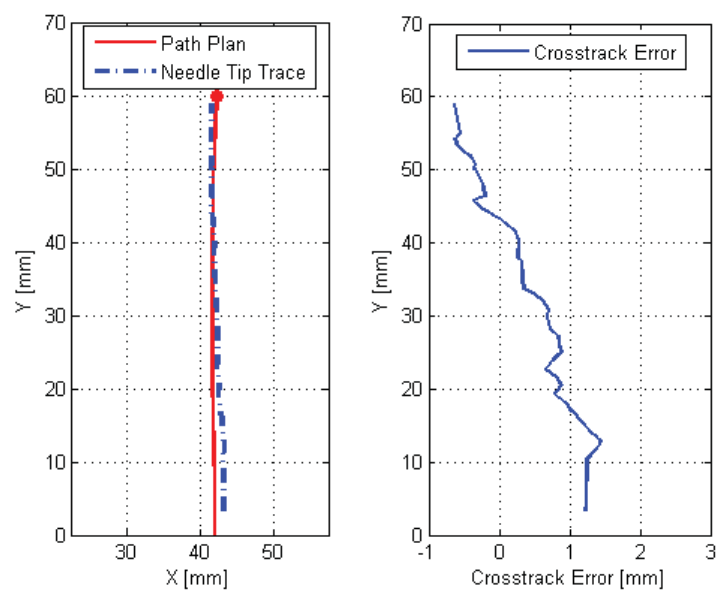

Figure 5. Simulated path tracking trace (left) and crosstrack error (right). Crosstrack error is the deviation of the needle tip from the path.

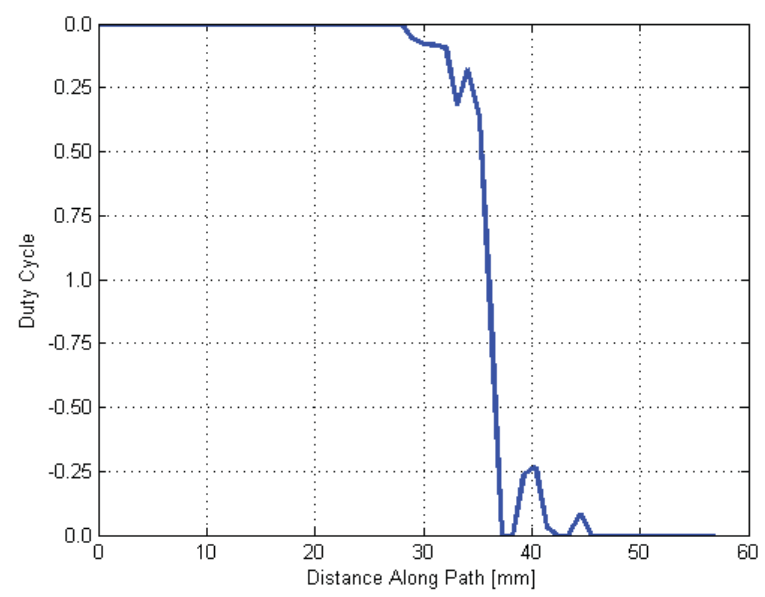

Figure 6. Path tracking control output from test in vitro. Duty cycles corresponding to maximum positive and negative curvature are located at the top and bottom of the vertical axis respectively. As the trace crosses a duty cycle of 1.0 curvature transitions from positive to negative. 


\section{DiscusSION}

These results show promise in closed-loop path tracking using needle steering via duty-cycled rotation for access to the kidney. In both simulation and testing in vitro, a needle was successfully driven along a pre-planned path in order to gain access to a kidney. Although the simpler unicycle model is used in the control derivation, the control law appears to work as intended.

Several matters are currently being addressed to further the work presented here. Refinement of the tracking algorithm, as well as improved pose estimation may drive the in vitro results to more closely match the simulated results. Look-ahead tracking algorithms and the full bicycle model are also being investigated. Also, in vitro testing using fluoroscopy as the imaging modality is planned to more closely simulate clinical application.

Extension of this work is necessary to control the needle in 3D. Methods currently under investigation include running a controller in parallel with $2 \mathrm{D}$ path tracking that constrains the needle in plane as well as the derivation of a 3D path following model.

In the longer term, needle designs which increase the range of motion while also limiting tissue damage will be vital for moving the technique toward clinical application.

\section{REFERENCES}

[1] V.G. Bird, B. Fallon, and H.N. Winfield, "Practice patterns in the treatment of large renal stones," J. Endourol., vol. 17, pp. 355-363, 2003.

[2] J.D. Watterson, S. Soon, and K. Jana, "Access related complications during percutaneous nephrolithotomy: urology versus radiology at a single academic institution," J. Urol., vol. 176, pp. 142-145, 2006.

[3] M.S. Michel, L. Trojan, and J.J. Rassweiler, "Complications in percutaneous nephrolithotomy," Eur. Urol., vol. 51, pp. 899-906, 2007.

[4] G. Hellawell, S. Mutch, G. Thevendran, E. Wells, and R. Morgan, "Radiation exposure and the urologist: what are the risks?," J. Urol., vol. 174, pp. 948-952, 2005.

[5] N. Abolhassani, R.V. Patel, and M. Moallem, "Needle insertion into soft tissue: a survey," Med. Eng. Phys., vol. 29, 2007, pp. 413-431.

[6] R.J. Webster, J.S. Kim, N.K. Cowan, G.S. Chirikjian, and A.M. Okamura, "Nonholonomic modeling of needle steering," Int. J. Robot. Res., vol. 25, pp. 509-525, 2006.

[7] D.S. Minhas, J.A. Engh, M.M. Fenske, and C.N. Riviere, "Modeling of needle steering via duty-cycled spinning," Proc. $29^{\text {th }}$ Annu. Int. Conf. IEEE Eng. Med. Biol. Soc., 2007, pp. 2756-2759.

[8] S. Thrun et al., "Stanley: the robot that won the DARPA Grand Challenge," J. Field Robot., vol. 23, pp. 661-692, 2006.

[9] V. Duindam, J. Xu, R. Alterovitz, S. Sastry, and K. Goldberg, "3D motion planning algorithms for steerable needles using inverse kinematics," Algorithmic Foundations of Robotics VIII, Springer Berlin / Heidelberg, 2009, pp. 535-549.

[10] L. E. Dubins, "On curves of minimal length with a constraint on average curvature, and with prescribed initial and terminal positions and tangents," Am. J. Math., vol. 79, no. 3, pp. 497-516, July 1957. 